agents followed by symptomatic medications of which decongestants were the most prevalent. On average, the number of drugs per prescription was 3.86. The combination of amoxicillin + clavulanate and azithromycin were the most prescribed antimicrobials.

Conclusions Overall, prescriptions of antimicrobials among pediatric patients suffering from RTIs were appropriate. There is scope for further improvement through formulation of institutional antibiotic guidelines for common RTIs.

\section{MULTIDRUG RESISTANT CLONES OF CA-MRSA ISOLATED FROM CHINESE CHILDREN AND THE RESISTANCE DETERMINANTS TO CLINDAMYCIN AND MUPIROCIN}

doi:10.1136/archdischild-2012-302724.0903

X Shen, L Wang. Beijing Children's Hospital, Capital Medical University, Beijing, China

This study aims to correlate the multidrug resistance (MDR) and ST clones of community-associated methicillin-resistant Staphylococcus aureus (CA-MRSA), to identify the genes responsible for clindamycin and mupirocin resistance in Staphylococcus aureus (SA) isolated from pediatric hospitals of Mainland China. Up to $435 \mathrm{SA}$ isolates were collected. The findings indicated that in comparison with community-associated methicillin-susceptible S. aureus (CA-MSSA), the resistance rates of CA-MRSA to ciprofloxacin, chloramphenicol, gentamicin and tetracycline were higher. The resistance rates to clindamycin and erythromycin were highe $(92.0 \%$ and $85.9 \%$, respectively) in CA-MRSA. The MDR rates were $49.6 \%, 100 \%$ and $14 \%$ in CA-MRSA, HA-MRSA and CA-MSSA isolates, respectively. Five of seven ST(sequence typing) clones in CA-MRSA, namely ST59, ST338, ST45, ST910 and ST965, had MDR rates of more than $50 \%(67.9 \%, 87.5 \%$, $100 \%, 50 \%$ and $83.3 \%$, respectively). The constructive phenotype of macrolide-lincosamide-streptogramin B(MLSB) resistance $(69 \%)$ and the ermB gene $(38.1 \%)$ predominated among them. The resistant rate to mupirocin was $2.3 \%$, and plasmids carrying the mupA gene varied in size between 23 and $54.2 \mathrm{~kb}$ in 6 strains with high-level resistance by Southern blot. The present study showed that the resistance to non- $\beta$-lactam antimicrobial agents, especially to clindamycin, was high in CA-MRSA isolated from Chinese children and the profile of resistance was related to clonal type.

\section{COMPARISON OF CLINICAL AND MICROBIOLOGICAL FEATURES OF VULVOVAGINITIS IN PREPUBERTAL AND PUBERTAL GIRLS}

doi:10.1136/archdischild-2012-302724.0904

A Yilmaz, N Celik, G Soylu, A Donmez, C Yuksel. Fatih University Medical Faculty, Ankara, Turkey

Objective Vulvovaginitisis the most common gynecological problem of childhood. The aim of the study was to determine and compare clinical and microbiological features of vulvovaginitis in prepubertal and adolescent girls.

Material and Methods In this retrospective study, the records of patients who were diagnosed with vulvovaginitis between January 2005 and December 2010 in the Pediatric outpatient clinic at Fatih University Hospital were retrieved. Information regarding age, symptoms, history of antibiotic use within 1 month prior to presentation, findings on urinalysis, serum antistreptolysin- $\bigcirc$ levels, and results of urine/vaginal cultures was collected.

Results The records of 112 patients were evaluated, 72 of which were prepubertal (64.2\%) and 40 were pubertal $(35.7 \%)$ at the time of diagnosis. Thirty-eight prepubertal patients $(52.7 \%)$ had a positive result on vaginal culture, the most commonly encountered microorganism being group A beta hemolytic streptococcus (15.2\%). Culture positivity rate in the pubertal group was $47.5 \%$ (19 patients), with Candida albicans being the most frequently isolated microorganism (27.5\%).

Conclusion The etiopathogenesis and culture results differ between prepubertal and adolescent girls with vulvovaginitis, which should be taken into consideration in the treatment approach of this disorder.

\section{INCIDENCE OF BRONCHIOLITIS HOSPITALIZATIONS IN THE PEDIATRIC TEACHING HOSPITAL ALCIDES CARNEIRO - PETRÓPOLIS - RIO DE JANEIRO - BRAZIL}

doi:10.1136/archdischild-2012-302724.0905

'EO Veiga, ${ }^{2} \mathrm{SA}$ Nogueira, ${ }^{2} \mathrm{SS}$ Cordeiro, ${ }^{2} \mathrm{FM}$ Moliterno, ${ }^{2} \mathrm{~A}$ Veiga, ${ }^{2} \mathrm{NV}$ Moliterno, 2RJ Silva, ${ }^{2} \mathrm{MPB}$ Souza. 'Paediatrics; ${ }^{2}$ Faculdade de Medicina de Petrópolis/HAC, Petrópolis, Brazil

Background and Aims The Bronchiolitis is the most common cause of hospitalization among infants under 6 months in developed countries and less than 1 year in developing countries. Respiratory syncytial virus is the main etiologic agent responsible for $60-80 \%$ of cases during the months of autumn and winter. The objective of this study was to describe the incidence of bronchiolitis in our country compared with the international literature.

Methods A transversal descriptive study of patients admitted to the pediatric ward of the Teaching Hospital Alcides Carneiro Petrópolis - RJ in the period June 2006 to June 2010 with clinical and radiological diagnosis of bronchiolitis. Excluding cases associated with underlying disease. We studied seasonal distribution, gender, age distribution, the most prevalent complications and hospital stay.

Results The bronchiolitis accounted for $5 \%$ of total admissions during the study period. Of these, $63 \%$ were male and mean age of 5.4 months in hospital stay of 7.5 days. Complications occurred in $48 \%$ of cases: pneumonia, atelectasis, $78.4 \%$ and $3.2 \%$. For fall-winter seasonality in $74 \%$ and $22.3 \%$ incidence in the month of May.

Conclusions This study demonstrates the seasonal nature and prevalence of males, age range and prevalence of pneumonia as the most frequent complication. The percentage of hospitalizations in this study in relation to the total number of hospitalizations is referenced by the above literature.

\section{FUNCTIONAL ANTIBODY ASSAY: HOW USEFUL IS IT IN RECURRENT RESPIRATORY TRACT INFECTIONS?}

doi:10.1136/archdischild-2012-302724.0906

DKK Ho, JJ Collier, M Abdelaziz, CM John. Department of Paediatrics, St Helens and Knowsley Teaching Hospitals NHS Trust, Liverpool, UK

Background and Aim Recurrent respiratory tract infection (RTI) is a frequent presenting complaint in the general paediatric clinic. Children are often tested to screen for possible underlying immunodeficiency and Cystic Fibrosis. We aim to evaluate the indications for performing functional antibody assay (FAA) and how the results affected our clinical management.

Methods We retrospectively studied children who had FAA (Haemophilus, Pneumococcus, and Tetanus) sent over a 1-year period in our district general hospital. Clinical records were examined for patient's characteristics and FAA results.

Results Between September 2010 and September 2011, 48 patients had FAA performed. Ages ranged between 1 and 18, with 6\% under 13 months old, and $85 \%$ under 8 years old. In $77 \%$, FAA was performed because of recurrent RTI; $10 \%$ because of other recurrent infections; and in $13 \%$ no indication was documented. $35 \%$ of 